\title{
'Social: An Algorithmic Stable Coin for Social Influence
}

\author{
By Andrew M. K. Nassief
}

\section{'Abstract}

Social is an algorithmic stable coin for social influence, built off of UMA or Universal Market Access Protocols, CloutContracts (CCS), and on the Celo network. It is based off of quantitative algorithmic stabilization provided by a social coin's perceived cryptographic value. Social is a utility and this technology is conceptual. How Social works, is by integrating statistical averages for CCS and social tokens staked within its network. It integrates universal market access in regards to CCS data (CloutContracts is a smart contracts platform for social influencers and creators). Social as an algorithmically pegged stable coin, will eventually create a standard for social influence. Decentralized finance applications might even peg cryptographic value to Social as a utility. Social can integrate decentralized oracles in order to process data much quicker over time, once the network becomes large enough. Since CloutContracts integrates social networks across various different places such as DeSo/BitClout, Minds, Peepeth, Steemit, etc., one can eventually establish some sort of cryptographic metric in regards to social influence, and develop complex algorithms centered around social physics and human behavioral processes. The same type of mathematical models that apply to quantitative algorithms in the stock market for example, can apply to social influence. The same also applies for mathematical models modeled after games like chess and go. Social as a stable coin, creates another complexity that CloutContracts can use to create new mathematical standards around market access data that it already has, and could be quite critical. Social influence as a market, and as some sort of utility, can then be looked at as either a metric, mathematical bet, or speculative model for new forms of political and human societies.

Keywords: Blockchain, Algorithms, Mathematics, Social Physics, Game Theory, Computational Sciences, Decentralization

\section{'Project Summary}

There are multiple components in regards to how Social will be constructed and what its core utility shall be. The first component is UMA or Universal Market Access [1]. Universal Market Access allows developers to easily create optimistic oracles and algorithmic models for forms of value. The second component in regards to how Social will be constructed is CCS or CloutContracts [2]. CloutContracts is a smart contracts platform for social media influencers, creators, and even blockchain engineers. It is oriented towards third party platforms. The third component is the Celo Network [3]. Celo is a mobile-first blockchain platform, whose transaction fees should allow for more adoptable uses. These are what will allow for Social to be developed in a scalable and robust way. Social will then be adoptable for others to use as some sort of standard as they wish (given it will be considered some sort of a stable coin). This shall open the door as well for many different usecases. 
' Core Features

In order to be adoptable, there are a series of core features in regards to Social and its core. Part of this is in regards to universal market access and optimistic oracles. The UMA protocol is currently compatible w/ Ethereum, but since CELO is EVM-compatible, likely some sort of implementation can take place either through direct or some sort of fork. An integration on top of Celo allows for easy adoptability given how expensive Ethereum gas fees are. The implementation of universal market access also helps create ways to accurate measure and implement data in relation to CloutContracts. This is important in regards to how Social will and can be used. It is also important for applications that integrate $\mathrm{w} /$ and around Social.

\section{'Premise}

The idea behind Social is to establish some sort of experimental market of social influence. These established markets centered around stores of cryptographic value can than eventually be mathematically modeled. Social, as an algorithmic and experimental stable coin, can be utilized in a variety of ways and a variety of metrics. Also, Social provides a valuable alternative to stable coins that may be viewed as being too centralized. Developers can build on top of Social, and it is pegged to tracking CloutContracts in a unique way as a utility. In order for Social to be massively useable and expandable, it needs to be built on top of a light network. Since, the specific data it is tracking is quite limited and it implements oracles, the network it needs to be on doesn't necessarily have to be as heavy as Ethereum or even a rollup layer. EVM-compatibility will likely be needed for sake of simplicity. Celo can be utilized given it is a light network, mobile-responsive, and EVM-compatible. However, Celo is a design choice. Things might and could change in the final implementation.

\section{'Usecases}

There are various usecases in regards to Social and possible technological integrations and applications. Some of these usecases include things such as Social as a utility or middleware, Social as a tool for algorithmic consensus, Social in regards to its utilization of market access data, and many others. Social eventually can be utilized as a metric in regards to perceived social influence value or perceived value of set amount of data. Over time, as more and more people utilize Social and CloutContracts becomes more and more popular, then the data points and perceived value of Social becomes more and more meaningful. The same concept applies in regards to usecases being built around or integrated with Social.

\section{'Implementation}

As of now, Social is conceptual. However, alot has been done in regards to conceptualization and technology. Deterministic Oracles and trainable/mineable algorithms can potentially be a huge aspect in regards to scaling Social. Social as mentioned utilizes a light network, is based off of UWA, and is tied to CloutContracts through the data it is analyzing. This is fundamental in regards to building it effectively. Also, most notably is given that Social is highly scalable, the technological implementation needing to start off as light, is crucial. Eventually, if sidechains, updated consensus, or even hard forks need to be done, this may be feasible in the future if cryptographic transaction fees are too high. 
'Technical Summary

Social is a(n) utility and algorithmic stable coin centered around the concept of social influence. Establishing a hypothetical value for social influence in cryptographic form, can also be utilized with various decentralized, blockchain-based or community-centered social networks. Networks such as Minds.com [4] for example, can eventually implement values in social influence on user's profiles (especially as more and more people implement CloutContracts as a utility). The same applies to other social networks as well, such as Peepeth [5]. With more usage, comes more data that is implemented in a meaningful, and decentralized manner.

'Impact

Most notably, Social does have the potential to disrupt multiple industries. For starters, measuring social influence can let us model different predictive markers that could be of value. Doing so in a way that is privacy-preserving or decentralized and in the blockchain is also a plus. Social allows influence and human-based decision making in regards to technological markets to be more in the hands of the people over centralized entities. Eventually, democratic consensus mechanisms can be formed. It goes way beyond just a stable coin in the cryptographic sense. The key aspect is the data behind it and the information being established over time.

\section{' Conclusion}

In conclusion, Social is an algorithmic stable coin pegged to CloutContracts in regards to its market access data as a utility. Eventually being able to establish $x$ value in social influence has many different usecases. Social will need to be built off of a large and scalable network that is also lightweight, Celo provides what may be quite a viable option.

' Disclaimer

Please keep in mind that CloutContracts and Social are utilities. The technology presented in this paper is conceptual or "as-is". None of this is meant to or warrants investment advice or financial information. The primary purpose of this paper is for educational and entertainment purposes.

' References

[1] Universal market access. UMA. (n.d.). Retrieved October 25, 2021, from https://umaproject.org/.

[2] Cloutcontracts. Home. (n.d.). Retrieved October 25, 2021, from https://cloutcontracts.net/.

[3] Mobile-first DEFI platform for fast, secure, and stable digital payments. Celo. (n.d.). Retrieved October 25, 2021, from https://celo.org/.

[4] Minds. (n.d.). The Leading Alternative Social Network. Minds. Retrieved October 25, 2021, from https://minds.com/.

[5] Microblogging with a soul (powered by blockchain). Peepeth. (n.d.). Retrieved October 25, 2021, from https://peepeth.com/welcome. 